\title{
Effectiveness of brief psychological interventions for suicidal presentations: a systematic review
}

\author{
Rose McCabe ${ }^{1}$, Ruth Garside ${ }^{2}$, Amy Backhouse ${ }^{1}$ and Penny Xanthopoulou ${ }^{1 *}$ (1)
}

\begin{abstract}
Background: Every year, more than 800,000 people worldwide die by suicide. The aim of this study was to conduct a systematic review of the effectiveness of brief psychological interventions in addressing suicidal thoughts and behaviour in healthcare settings.

Methods: Following PRISMA guidelines, systematic searches were conducted in MEDLINE, CINAHL, EMBASE, the Cochrane Central Register of Controlled Trials and PsycINFO databases. A predefined search strategy was used. Two independent reviewers screened titles and abstracts followed by full texts against predefined inclusion criteria. Backward and forward citation tracking of included papers was conducted. Quality appraisal was conducted using the Cochrane Risk of Bias Tool for Randomized Controlled Trials and the CASP tool for randomised controlled trials. The small number and heterogeneity of studies did not allow for meta-analysis to be conducted. A narrative synthesis was conducted.

Results: Four controlled studies of brief psychological interventions were included, conducted in Switzerland, the U.S. and across low and middle-income countries. Three studies were conducted with adults and one with adolescents. All studies were judged to be at low risk of bias. All of the interventions were implemented with patients after attending emergency departments and involved 3412 participants. The main outcomes were suicide, suicide attempts, suicidal ideation, depression and hospitalization. The components of the interventions were early therapeutic engagement, information provision, safety planning and follow-up contact for at least 12 months. The interventions drew to, different degrees, on psychological theory and techniques. Two trials that measured suicidal ideation found no impact. Two studies showed fewer suicide attempts, one showed fewer suicides and one found an effect on depression.
\end{abstract}

Conclusions: Although the evidence base is small, brief psychological interventions appear to be effective in reducing suicide and suicide attempts. All studies to date have been conducted with people who had attended the ED but the interventions could potentially be adopted for inpatient and other outpatient settings. Early engagement and therapeutic intervention based on psychological theories of suicidal behaviour, sustained in follow-up contacts, may be particularly beneficial.

Trial registration: Systematic review registration: PROSPERO CRD42015025867.

Keywords: Suicide, Suicidal ideation, Systematic review, Controlled studies, Effective communication

* Correspondence: p.d.xanthopoulou@exeter.ac.uk

${ }^{1}$ University of Exeter Medical School, Heavitree Road, Exeter EX1 2LU, UK

Full list of author information is available at the end of the article 


\section{Background}

Suicide is a serious public health concern with more than 800,000 deaths from suicide every year worldwide [1]. This is one suicide every 40 seconds [2]. Suicide prevention is a global public health priority.

Certain groups have a higher risk of suicide. The majority of deaths by suicide (78\%) occur in low and middle-income countries. There are also significant gender differences with men more likely to die by suicide (male-to-female ratio 1.7 in 2015) [1]. Younger people are also more likely to die by suicide: $55 \%$ of deaths by suicide are among the 15-44 age group with suicide ranked as the second leading cause of death among 15-29 year-olds [1].

Many people who take their own life have been in contact with healthcare professionals in acute hospitals and/or primary or secondary care before they die. In the U.K., $40 \%$ of people attended the general emergency department in the year before death, having attempted suicide [3]. Around one in four people who take their own life have been in contact with mental health services the year before death in the U.K. and around one in three in the U.S. [4]. Meanwhile, $45 \%$ of people who take their life were seen in primary care the month before death in the U.K. with a similar figure of $47 \%$ in the U.S. [4].

Collectively, this is a very high number of face-to-face contacts between healthcare professionals and people who go on to take their own life. Referring patients to specialist services is often not a realistic option because they are not available or where they are available, there is not enough capacity in these services. Specialist treatment is very costly and many patients do not attend or drop out of treatment prematurely [5]. Hence, in routine contacts with people at risk of suicide, there is potential for brief therapeutic interventions. There are longer term psychological interventions (e.g. dialectical behaviour therapy, cognitive behaviour therapy) to address suicide [6, 7] and self-harm [8]. However, it is not clear if limited brief interventions that can be administered in routine frontline encounters where healthcare professionals encounter people at risk of suicide can be effective [9, 10].

Studies in healthcare and other settings (e.g. educational) $[11,12]$ generally report brief interventions as lasting 1-3 sessions [13, 14]. We focused on brief interventions as they are more likely to be integrated into routine clinical practice without the need for significant additional resources or extensive reconfiguration of existing services. Brief interventions that could be deployed in routine care, rather than referring people to another service, are of particular interest as they could be deployed at scale to improve patient outcome.

\section{Objectives}

The aim of this review was to evaluate the effectiveness of brief psychological interventions to address suicidal thoughts and plans, focusing on two objectives:

1. To identify controlled studies of brief psychological interventions to address suicidal thoughts and plans in healthcare settings.

2. To describe the interventions used by professionals/ paraprofessionals that are effective in addressing suicidal thoughts and plans.

\section{Methods}

\section{Protocol and registration}

Approaches to searching, methods of analysis and inclusion criteria were specified in advance and documented in a protocol [15], with some changes made in the course of the study (recorded on PROSPERO: CRD42015025867), relating mainly to the eligibility criteria. The PRISMA standards of quality for reporting meta-analyses [16] were used to plan, conduct and report this review.

\section{Eligibility criteria}

The review included published controlled studies that reported on brief psychological interventions to address suicidal thoughts and plans in healthcare settings.

\section{Inclusion criteria}

Participants Participants of any age and gender at risk of suicide.

Interventions Brief interventions delivered in any healthcare setting to the specified population:

- Interactions between professionals/paraprofessionals (e.g. lay mental health workers, nursing assistants, educators, volunteers) and patients

- Addressing suicidal thoughts and plans

- Two-way communication (i.e. not one-way communication in the form of letters/postcards/text messages or exclusively self-guided questionnaires/ instruments) between at least one professional/paraprofessional and one patient; other people can be present

- Focus on suicidal thoughts and plans rather than diagnostic conditions, e.g. depression, anxiety, borderline personality disorder

- Focus on routine clinical encounters

- Brief interventions, defined as up to three sessions delivered in/soon after presenting episode, which can be supplemented by further follow-up contact 
Comparator Any comparison or no comparator/usual care.

Outcome measures Primary outcome was suicidal ideation, using any measure. Other outcomes included: Identification of suicide risk, suicide attempts, suicide, hope, patient distress and depression. Suicidal ideation is defined according to Beck's 'Scale for Suicide Ideation' [17] as the intensity of current conscious suicidal intent, examining various dimensions of self-destructive thoughts or wishes.

Types of studies No restrictions were placed on study location or publication date of included studies. We included cluster randomised controlled trials, randomised controlled trials, controlled before-and-after studies and controlled pre-test/post-test designs. We excluded noncontrolled studies.

Exclusion criteria Assisted suicide; Self-harm without intent to die, i.e., direct, deliberate destruction of one' $\mathrm{s}$ own body tissue in the absence of intent to die, which differs from suicide attempts with respect to intent, lethality, chronicity, methods, cognitions, reactions, aftermath, demographics and prevalence [18].

\section{Search and information sources}

Database searches were conducted from date of inception to June 2015, and updated in August 2016 and in April 2017. The following databases were searched: MEDLINE in Process (Ovid), PsycINFO (Ovid), EMBASE (Ovid), The Cochrane Central Register of Controlled Trials (CENTRAL) (Wiley Online Library) and CINHAL (EBSCO). Trial registers (ISRCTN registry, ClinicalTrials.gov) were searched for published and ongoing trials, references of previous systematic reviews were searched and experts in the field were contacted in order to identify any new studies.

The search strategy is presented in Additional file 1. Suicide, study design and communication/interaction terms were combined using Boolean logic (AND, OR) and specific tested filters were used for study design (The InterTASC Information Specialists' Sub-Group filters). Medical Subject Heading (MeSH) terms were also used. EndNote X7.0.2 software was used to manage searches and references.

\section{Study selection}

Search results were exported to EndNote and duplicates were automatically identified and removed. Records that were not removed automatically we identified and removed by hand. Two independent reviewers (PX, RM/ $\mathrm{AB})$ were involved in screening all titles and abstracts, full paper screening, quality appraisal and assessment of risk of bias of included studies. Disagreements or uncertainties were discussed in meetings and email correspondence between all authors.

\section{Data extraction}

We developed a data extraction form based on the Cochrane Risk of Bias Tool for Randomized Controlled Trials, which we modified to reflect the diversity of included studies. The extraction form was piloted (RM, $A B, P X)$ before being finalised. Data was extracted by one author (RM/PX) and checked by another (RM, $A B$, $\mathrm{PX})$. The authors of three included studies were contacted to obtain additional data, trial protocols and further detail on the relevant intervention. Additional information was also obtained from other publications reporting on the study [19].

\section{Risk of bias}

Risk of bias was assessed using the Cochrane Risk of Bias Tool for Randomized Controlled Trials on 6 criteria. Each criterion was rated as low, medium or high. Using these ratings, we generated an overall risk of bias score by scoring the ratings on the first 5 criteria: sequence generation, allocation concealment, blinding, incomplete outcome data and selection bias. A score of 3 was allocated to a 'low' risk, a score of 2 was allocated to 'medium' risk and a score of 1 was allocated to 'high' risk. The total score could range from 5 to 15 , with a higher score indicating lower risk of bias.

Study quality was also assessed using the CASP (Critical Appraisal Skills Programme) for randomised controlled trials checklist [20]. Two raters independently assessed the risk of bias for each study (PX and RM/ $\mathrm{AB})$. The individual items on the score sheets were then checked by three authors ( $\mathrm{RM}, \mathrm{AB}, \mathrm{PX})$ in an interreviewer discussion where disagreements were resolved.

\section{Analysis}

The studies were too heterogeneous to combine in a meta-analysis, in terms of the outcomes they measured. Hence, a narrative synthesis [21] was conducted. This involved developing a preliminary synthesis, focusing on the outcomes, interventions and heterogeneity across the studies, followed by iteratively exploring relationships in the data, contexts of the interventions and mechanisms for change, using visual representations (tables) [21]. Where not available, relative risk was calculated using the MEDCALC relative risk statistical calculator (https://www.medcalc.org/calc/relative_risk.php).

\section{Results}

Study selection

After removing duplicates, a total of 17,201 titles and abstracts were identified and screened (Fig. 1). Of these, 44 


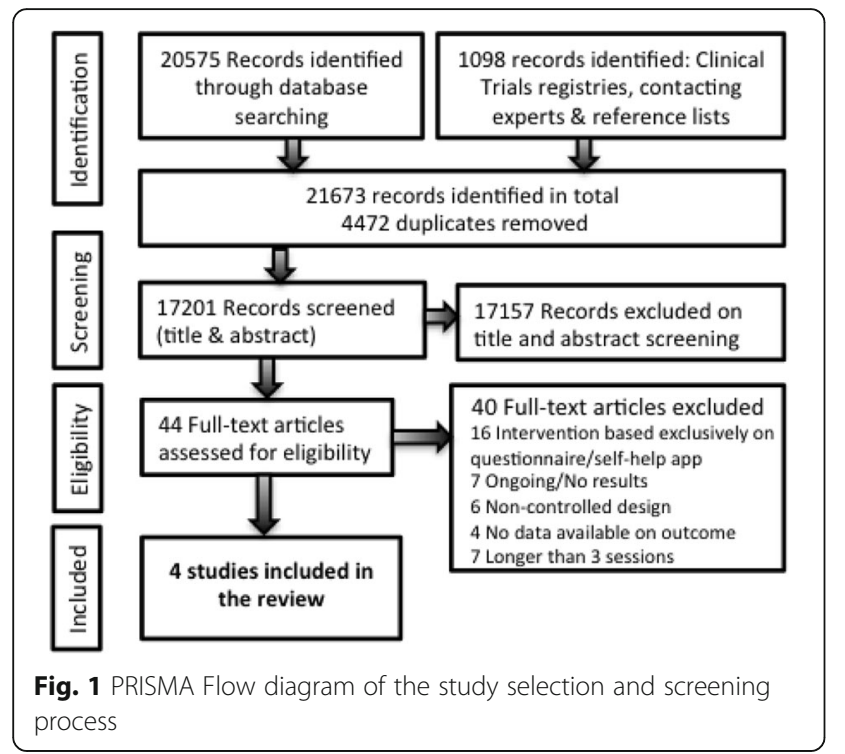

full-text articles were assessed for eligibility. Forty fulltext articles were excluded studies due to a lack of control in the study design, no data available on treatment outcome, interventions were exclusively based on questionnaires or longer than three sessions. Four studies met the inclusion criteria and were included in the review.

The included studies encompassed two RCTs, one pilot RCT and a quasi-experimental study. All reported on interventions in the emergency department setting. The non-randomised controlled study used an interrupted time series design [22] and the three RCTs involved individual patient randomization [23-25]. The interventions were compared to treatment as usual (TAU) [23] and enhanced TAU [22, 24, 25].

\section{Risk of bias}

The risk of bias assessment, using Cochrane Risk of Bias Tool for Randomized Controlled Trials, is presented in Fig. 2. The overall score for each study (see Methods section for scoring) was: Fleischmann [23] 14 out 15, Gysin-Maillart [24] 13 out of 15, King [25] 13 out 15, and Miller [22] 10 out 15.
Three studies were of high quality. High/medium risk of bias was reported for blinding professionals across all studies, however, it would not have been possible to blind professionals as they were delivering the interventions. One study [22] presented medium risk of bias, where lower scores related to not using randomization to allocate interventions to participants (the study employed an interrupted time series design [26]).

Studies rated high in the CASP for randomised controlled trials checklist (results are presented in Additional file 2).

\section{Characteristics of participants and outcomes}

In total, the studies included 3412 participants (range 49-1867). Three studies included adult suicide attempters [22-24] and one [25] focused on adolescents with suicide risk factors (e.g. depression and alcohol misuse). Study characteristics are presented in Table 1.

As there were only four studies that differed in what outcomes they assessed and when these outcomes were assessed (2, 12, 18 and 24 months), a meta-analysis was not appropriate. One study was conducted across 5 countries and the included paper reports results across all 5 sites. Separate results for one of the sites (Iran) are reported elsewhere [27], however we did not include this study due to overlapping data.

\section{Characteristics of interventions}

The interventions focused on engagement, safety planning, information and follow-up contact after discharge from the emergency department. The duration of the interventions ranged from 12 to 24 months.

The four interventions in the included studies were:

a) brief intervention and contact (BIC) [23]

b) the attempted suicide short intervention program (ASSIP) [24]

c) teen options for change (TOC) [25]

d) Safety Assessment and Follow-up Telephone Intervention (SAFTI) [22]

\begin{tabular}{|c|c|c|c|c|c|c|}
\hline & $\begin{array}{l}\text { Sequence } \\
\text { generation }\end{array}$ & $\begin{array}{c}\text { Allocation } \\
\text { concealment }\end{array}$ & $\begin{array}{l}\text { Blinding of } \\
\text { participants, } \\
\text { personnel and } \\
\text { outcome } \\
\text { assessors }\end{array}$ & $\begin{array}{l}\text { Incomplete } \\
\text { outcome } \\
\text { data }\end{array}$ & $\begin{array}{l}\text { Selective } \\
\text { outcome } \\
\text { reporting }\end{array}$ & $\begin{array}{l}\text { Other } \\
\text { sources of } \\
\text { bias }\end{array}$ \\
\hline Fleischmann et al. & Low & Low & Medium & Low & Low & Unclear \\
\hline Gysin-Maillart et al. & Low & Medium & Medium & Low & Low & Low \\
\hline King et al. & low & Low & High & Low & Low & Unclear \\
\hline Miller et al. & High & High & Medium & Low & Low & High \\
\hline
\end{tabular}

Fig. 2 Risk of bias assessment (Cochrane Risk of Bias Tool for Randomized Controlled Trials) 


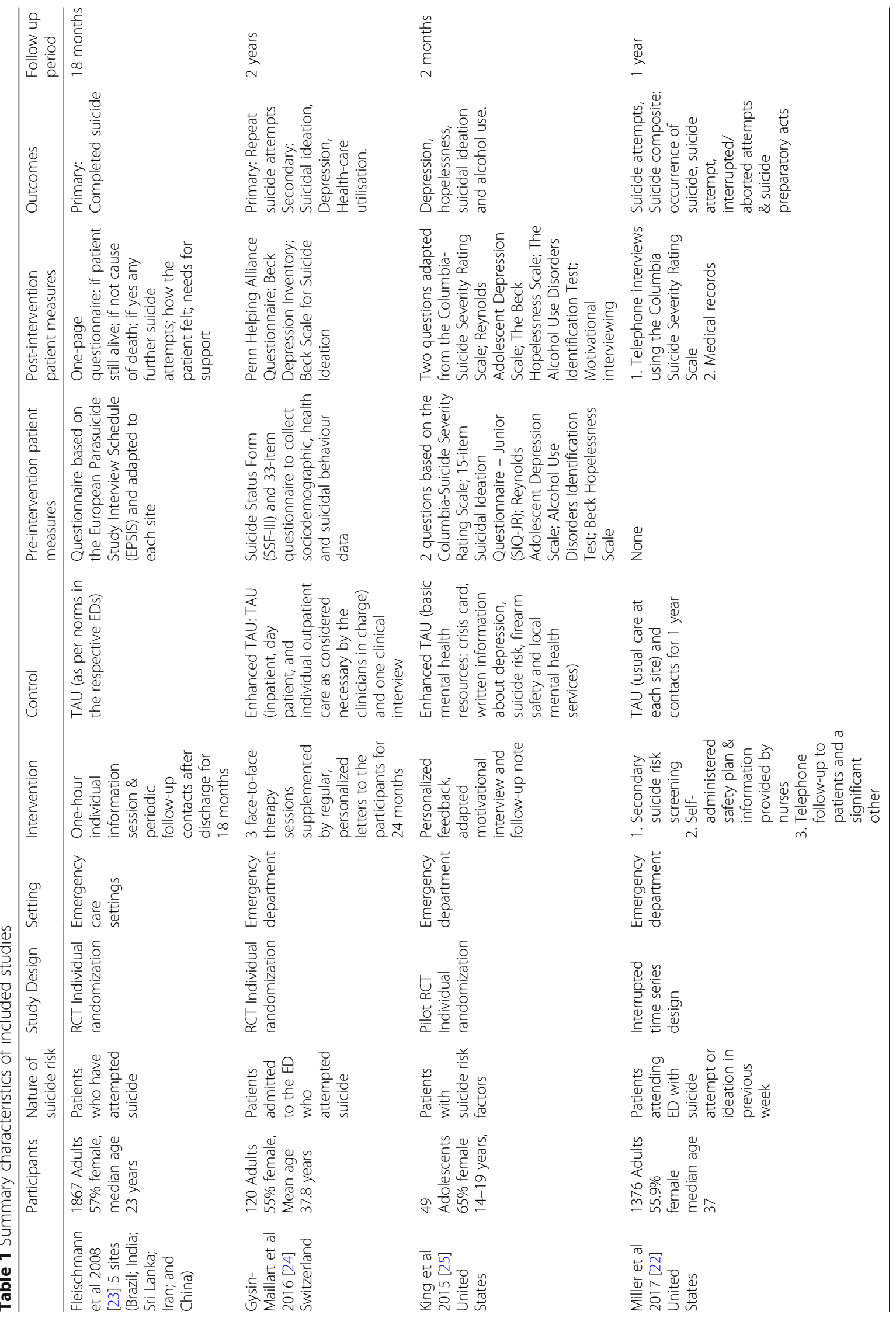


A summary of the interventions is presented in Table 2 . The three larger studies [22-24] used 1-3 individual sessions soon after discharge from the ED and follow-up contacts over 18, 24 and 12 months respectively. The interventions varied according to when the intervention starts, whether patients are seen soon after discharge from the ED and then how often they are contacted during the follow-up period.

Gysin-Maillart [24] implemented a therapeutic intervention focused on engaging the person in a narrative interview about the suicidal crisis in a first session soon after the ED attendance. This then progressed to case conceptualization and individualized safety planning in another 2 sessions. Then, patients were contacted via letter for 24 months, every 3 months in the first year and every 6 months in the second year. Fleischmann [23] implemented a single information session to understand and manage suicidal behaviour followed by up to 9 phone calls or visits over 18 months. In the trial by Miller et al. [22], the intervention consisted of secondary suicide screening, information provided by nurses, a self-administered safety plan and up to 7 brief (10$20 \mathrm{~min}$ ) calls to the patient and up to 4 calls to a significant other.

\section{Theoretical rationale and aims of the interventions}

The interventions, to varying degrees, focus on informing people about suicidal behaviour, helping people to become aware of problems/vulnerability/events linked to the behaviour, exploring ambivalence and motivating people to engage in safety planning and help-seeking, problem solving and developing practical strategies to manage future suicidal crises along with signposting to helplines/professionals.

Two interventions (BIC, ASSIP) foreground the role of the relationship: BIC follow-up contacts aim to give patients a feeling of being seen and heard by someone. ASSIP aims to establish an early therapeutic alliance to maximise engagement in treatment, with the follow-up contact reinforcing the relationship.

While all of the interventions comprise information, safety planning and follow-up contact, they varied in the extent to which they used psychological theories and techniques. Gysin-Maillart's and King's interventions were based more on psychological theories (i.e. action theory and theory of health behaviour) and techniques than Fleischmann and Miller.

\section{Completion of the intervention}

Completion of the intervention ranged from $60.8 \%$ to 93\% across studies. In the Fleischmann trial, it appears that $91 \%$ received the intervention. In the Gysin-Maillart trial, $93 \%$ completed the intervention. $85 \%$ of patients in the King trial received the full intervention. Miller et al. reported that $60.8 \%$ received at least part of the intervention (i.e. 1 telephone call).

\section{Completion of outcome assessments}

Fleischmann reported a 9\% loss to follow-up at 18 months. Gysin-Maillart reported a $14 \%$ loss to follow-up at 24 months. King reported low loses (6\%), however this was for a very short follow up period of 2 months. Miller et al. reported that assessment of suicide attempts was conducted for all participants during the 52-week follow-up period, whereas 20\% (1089 of 1376 enrolled participants) did not have a suicide composite outcome, which was derived from the telephone interview (self-reported) at 52 weeks.

\section{Effectiveness of interventions}

Brief psychological interventions were effective in reducing suicide, suicide attempts and depression (see Table 3). Interventions used a range of methods to measure these outcomes.

\section{Suicidal ideation}

Two studies found no effect for suicidal ideation [24, 25].

\section{Suicide}

One trial was effective in reducing suicide over 18 months, with a $90 \%$ relative risk reduction in completed suicides [23] $(\mathrm{RR}=0.10,95 \% \mathrm{CI} 0.02$ to 0.45 , $p=0.0025$ ).

\section{Suicide attempts}

Two studies reported an effect for repeat suicide attempts. Miller [22] reported a relative risk reduction of $20 \%$ for the intervention phase (RR 0.80, 95\% CI 0.63 to 1.02 ). GysinMaillart [24] reported a mean hazard ratio of 0.17 (95\% CI $0.07-0.46$ ), indicating that the ASSIP group had an $83 \%$ reduced risk of attempting suicide during the 24-month follow-up period compared to the control group (Wald $x^{2}$ $1=13.1$, 95\% CI 12.4-13.7, $p<0.001)$. They also conducted an analysis removing those with BPD and found that when individuals with BPD were excluded, the ASSIP group had an $89 \%$ lower risk of attempting suicide (mean hazard ratio of 0.11 (95\% CI 0.03-0.49)).

Miller [22] also reported an effect for a suicide composite' measure (RR $0.85,95 \%$ CI 0.74 to 0.97 ), which measured 5 types of suicidal behavior: death by suicide, suicide attempt, interrupted or aborted attempts, and suicide preparatory acts.

\section{Depression}

Of the two studies assessing depression, one study by King [25] found a significant effect, however another by Gysin-Maillart [24] did not. King focused on adolescents 


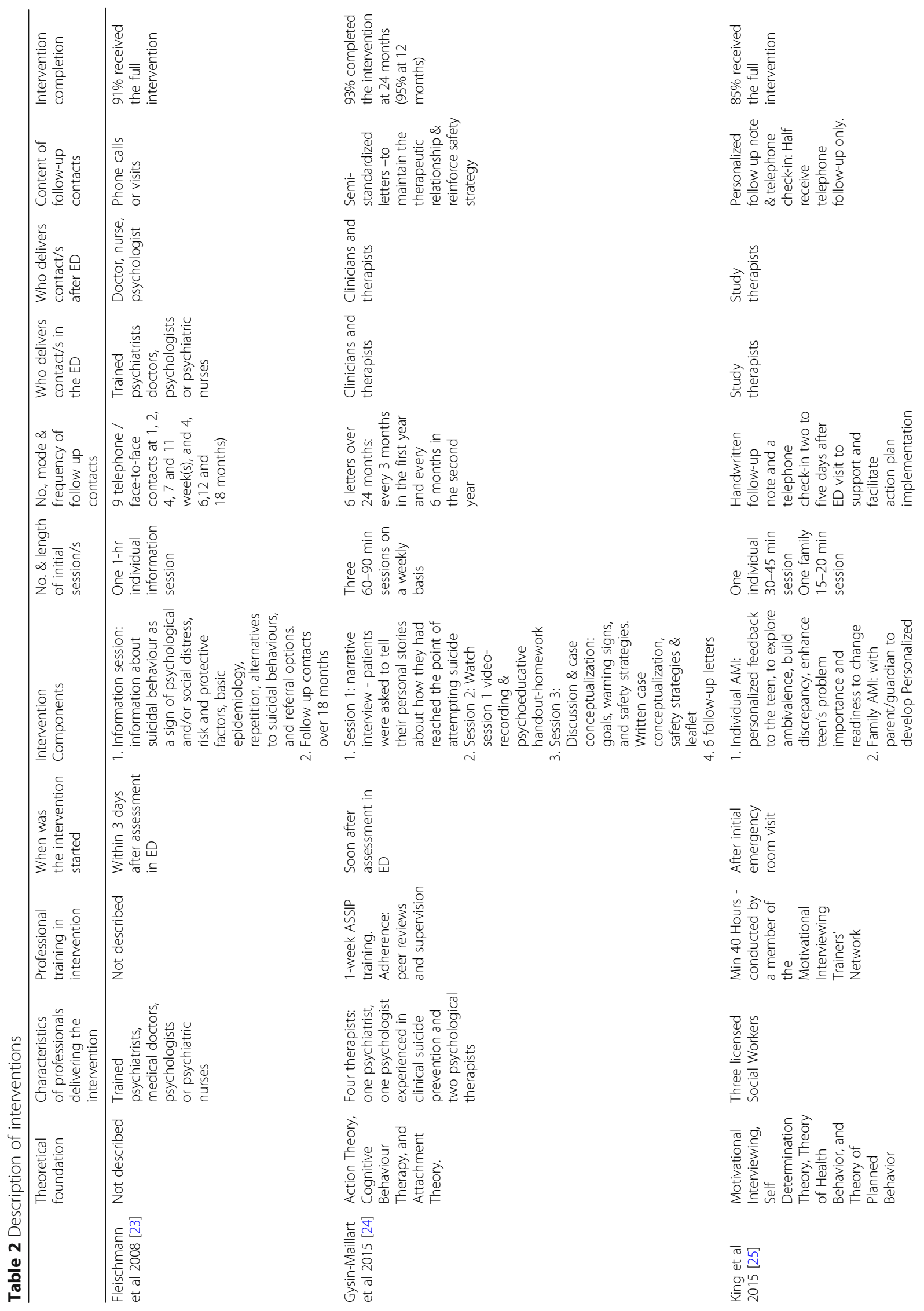


McCabe et al. BMC Psychiatry (2018) 18:120

Page 8 of 13

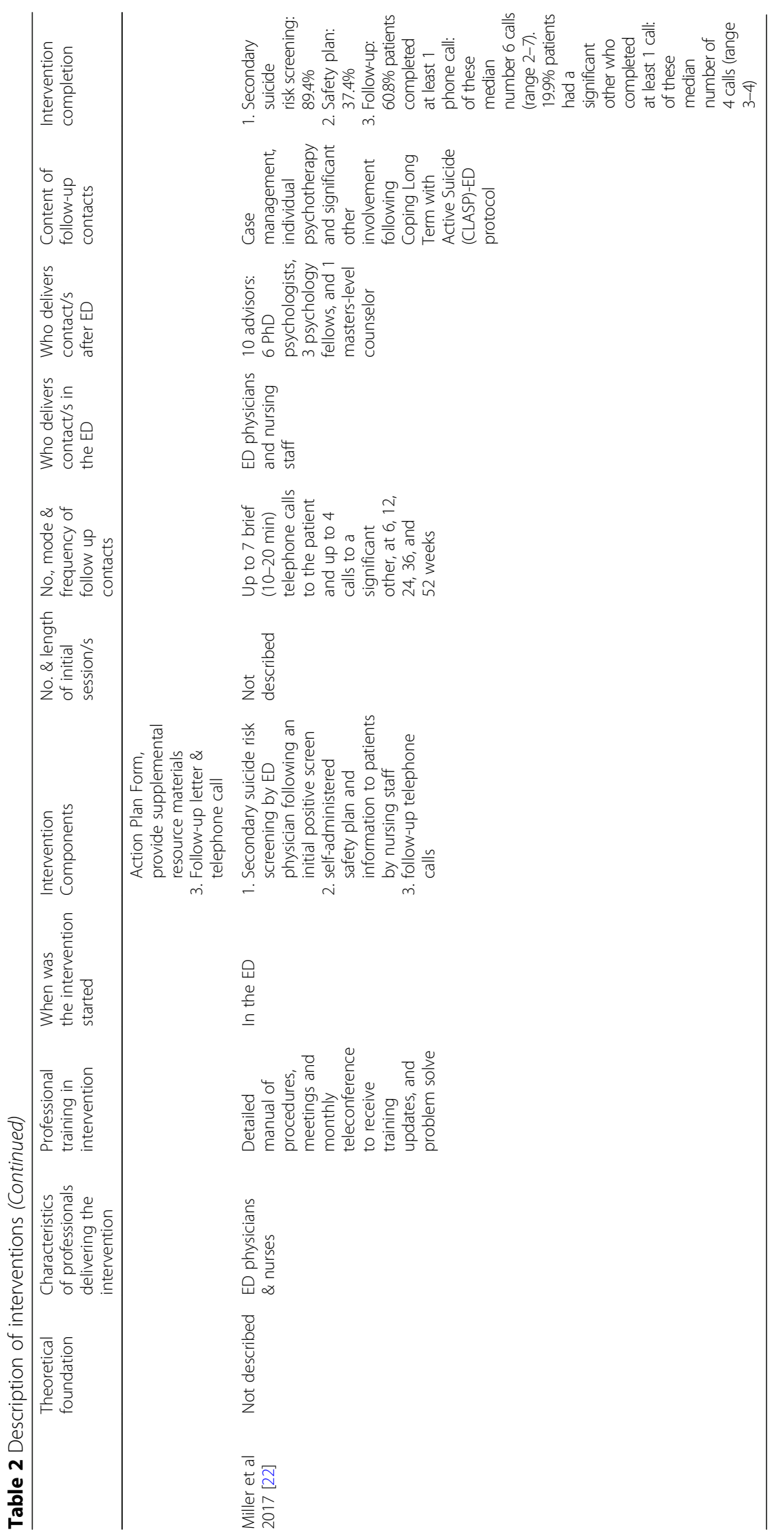




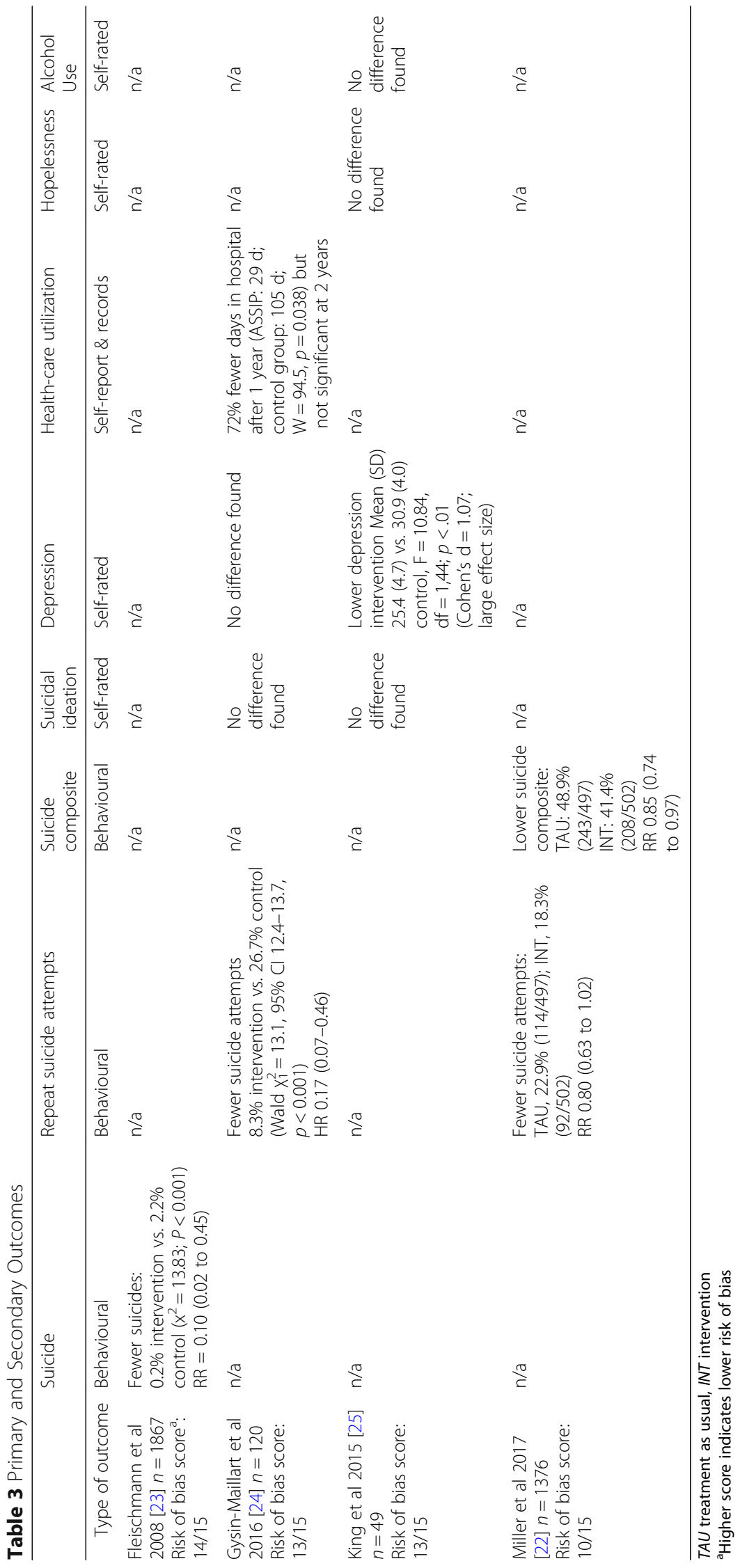


over a shorter follow-up period of 2 months while Gysin-Maillart focused on adults (with longer-standing difficulties) over a longer follow-up of 2 years.

\section{Healthcare use}

Gysin-Maillart [24] found a significant reduction in hospitalization, with $72 \%$ fewer days in hospital over 1 year, which was no longer significant after 2 years $(p=0.08)$.

\section{Alcohol use and hopelessness}

Where assessed [25], no effect was found for alcohol use and hopelessness.

Analysis Miller [22] and Gysin-Maillart [24] used intention to treat analysis. However, Fleischmann [23] did not: they analysed the participants who were not lost to follow-up which corresponded to $91 \%$ of the sample. King's [25] analysis of intervention effect used per protocol, rather than intention to treat, analysis. We cannot tell the direction or magnitude of impact of this, but there was little loss to follow-up ( $<10 \%$ LTFU).

\section{Discussion}

Four controlled studies of brief psychological interventions to reduce suicidal behaviour and suicide were identified, three with adults and one with adolescents. All of the interventions were implemented with patients who had attended the ED and involved a total of 3412 participants. The interventions had three common components, namely information about/understanding of the suicidal crisis, safety planning and follow-up contact along with different degrees of psychological input. One (out of one study assessing suicide) found fewer suicides [23]. One (out of one study) assessed a 'suicide composite' score [22] and found a lower suicide composite score. Two (out of two studies assessing suicide attempts) found fewer suicide attempts [22, 24]: Miller found a small but meaningful difference with a number needed to treat of 22 and Gysin found an $83 \%$ reduced risk of attempting suicide during the 24-month follow-up period. Two (out of two) studies measuring suicidal ideation did not show an effect $[24,25]$. One (out of two studies measuring depression) found an improvement in depression [25]. One (out of one study measuring hopelessness) found no improvement in hopelessness [25]. One (of one study assessing hospitalisation) found $72 \%$ fewer days in hospital after 1 year but no significant difference after 2 years [24]. Hence, there appear to be greater changes in behavioural outcomes than in symptom outcomes, suggesting that patients may still be experiencing suicidal ideation but make fewer suicide attempts and are less likely to die by suicide.

One trial [23] found an effect on suicide, which was conducted across 5 low and middle-income countries. The authors concluded that a brief intervention was likely to have reduced suicide by providing a social support network for people with limited social support in countries with modest infrastructure and financial/human resources. Two trials found an effect on suicide attempts. One was a large trial in the U.S. with 1376 participants [22] and one a small trial in Switzerland with 120 participants [24]: the large trial found a $20 \%$ relative risk reduction and the smaller trial with a $83 \%$ relative risk reduction. The large trial focused on information provided by nurses and a self-administered safety plan in the ED, followed by 7 telephone calls to the patient and 4 calls to a significant other over one year. Meanwhile, the smaller trial demonstrating the larger effect, focused on 3 face-to-face therapeutic sessions soon after discharge from the ED and follow-up letters over 24 months.

Given the low prevalence of suicide as an outcome, studies in this area use various proxy and composite measures. One study reported on completed suicide, two studies reported on suicide re-attempts and only one study reported healthcare utilisation (i.e. hospitalisation). Suicide attempts were measured using hospital records, however Miller also used telephone interviews to collect information on this outcome, which could address some of the issues of reliability and accuracy of hospital records. This area would benefit from more RCTs with larger populations, that report on completed suicide [6].

What might explain the large effect in the smaller trial? The two trials recruited participants with a similar age range (mean $=37.8$ in Gysin's smaller trial, and median $=37$ in Miller's larger trial) and male-tofemale ratio (Gysin 55\% female, Miller 56\% female). However, the smaller trial [24] was conducted in one ED while the larger trial [22] was conducted across 8 EDs so local championing and fidelity to the intervention may have been stronger in the single site smaller trial. In addition, the smaller trial involved more intensive psychological input with an emphasis on an early therapeutic alliance in face-to-face sessions along with follow-up contact by the same rather than different professionals. A better therapeutic alliance was associated with a lower rate of suicide attempts [28], suggesting that early engagement and therapeutic intervention soon after the ED attendance may be particularly beneficial.

The interventions varied on some important factors, most notably the psychological theories underpinning the intervention, the intensity of and the proposed mechanisms and wider socioeconomic context of the intervention. These were to some extent reflected in when, how and by whom the initial and follow-up contacts were made and what happened in these contacts. The BIC intervention leading to fewer deaths by suicide in low/middle-income countries focused on information, practical advice and signposting and was delivered by 
doctors, psychologists or nurses. Interventions leading to fewer suicide attempts in countries where better mental health services exist were based more on psychological theories underpinning suicidal behaviour $[24,25]$ and psychological techniques to explore motivation for change and safety strategies delivered by trained clinicians or therapists $[24,25]$. These differences are consistent with realist evaluation [29] pointing to what works in which circumstances and for whom. In three studies, follow-up contact was over the telephone. This makes interventions more viable and cost-effective when resources are scarce while also allowing for flexibility and improved access to treatment when, for example it might be geographically unavailable [30].

Similar to a previous review of suicide interventions [7], the contribution of the individual components of the interventions is unclear as the interventions were evaluated as a whole. Moreover, it is not clear what the contribution of more frequent contacts is and up until which point these contacts are optimally effective.

One of the four studies was conducted in low and middle-income countries, which has implications for the generalizability of the results to countries with stronger health and social care systems. Treatment as Usual is described in the studies as usual care in clinical practice. This is likely to have varied considerably as the studies were conducted in different countries with different healthcare systems. For example, this consisted of inpatient, day patient and outpatient care in the study by Gysin in Switzerland. However, this is likely to have been considerably less ("as per the norms in the respective EDs") in the Fleischmann study which was conducted across 5 different low and middle-income countries. This introduces considerable heterogeneity in interpreting the findings.

All of the studies evaluated interventions that were implemented with people after attending the ED, with two interventions explicitly also involving family or significant others [22, 25] Two studies focus on high risk populations, i.e., people in low and middle-income countries [23] and young people [25]. The ED setting is particularly important as a large number of at-risk individuals use emergency services [31]. People who attend the emergency department are at high risk of a further suicide attempt, with studies showing that around $20 \%$ re-present within one year [31]. It is estimated that hospitals in England manage over 200,000 episodes of self-harm each year. Many people who attend the ED in a crisis do not attend specialist mental health services for follow-up. Hence, brief ED interventions to reduce suicide risk may be especially useful [32]. Although the identified studies were conducted in the ED, the interventions - as a whole or components of the interventions - could be tested in other treatment settings such as inpatient or outpatient community treatment settings.

Strengths and limitations This review used a systematic approach to identify controlled studies of brief psychological interventions for suicidal thoughts and behaviour. It identified the usefulness of brief interventions to address suicidality in the ED. However, and as previously found [11], the evidence base is small. As is commonly found in systematic reviews with a limited evidence base, the included studies were disparate in their design, outcome measurement tools, measurement intervals and types of interventions offered. As they assessed different outcomes at differing time points, a meta-analysis was not appropriate. In addition, one of the four studies was conducted in low and middleincome countries, which has implications for the generalizability of the results. The findings from this study may not be generalizable to higher income countries with stronger health and social care systems. However, the narrative synthesis allowed us to summarise the state of the art across somewhat heterogeneous studies. Detailed information was lacking on specific aspects of the intervention (e.g. length of components and randomisation procedure) in some studies, which restricted the interpretation of the methodological quality. Risk of bias may be reduced if those assessing outcomes are blinded to treatment allocation. However, as some of the outcomes are objective, such as suicide [23], these are not subject to bias. With suicide attempts, there is some element of judgment but, if assessors are blinded, there is less chance of bias. Assessors were blinded in Miller and King but not in Gysin. Suicidal ideation is self-rated and so cannot be blinded. Finally, as they are receiving a psychological intervention, it is not possible to blind participants to treatment allocation.

The review focused on brief interventions that aimed to enhance treatment. While, these interventions often included considerable follow-up contact, we did not include studies solely focusing on follow-up contact. For example, a study in France consisting of a single telephone contact one month after attending the ED was categorized as a follow-up intervention rather than enhancing the index treatment episode/s [33].

\section{Conclusions}

Although there are relatively few studies to date, brief psychological interventions appear to be effective in reducing suicide and suicide attempts. However, it is unclear to what extent the effect is due to specific psychological techniques/components or to more frequent contacts, which should be investigated in future 
studies. All studies to date have been conducted in the ED. The interventions tested do not appear to reduce suicidal ideation, suggesting that although patients may still be in considerable distress, the interventions affect change in behaviour, i.e., fewer suicide attempts and suicides, by targeting information and understanding about the suicidal crisis, safety planning for future crises and follow-up contact to monitor and support patients. Early engagement and therapeutic intervention based on psychological theories of suicidal behaviour, sustained in follow-up contacts, may be particularly beneficial.

\section{Additional files}

Additional file 1: Search strategy. Predefined search strategy developed in Ovid MEDLINE(R) 1946 to Present. (DOCX 14 kb)

Additional file 2: Critical Appraisal Skills Programme (CASP) for a Randomised Controlled Trial. CASP for randomised controlled trials checklist. (DOCX $22 \mathrm{~kb}$ )

\section{Abbreviations}

ASSIP: Attempted suicide short intervention program; BIC: Brief intervention and contact; CASP: Critical appraisal skills programme; ED: Emergency department; RCT: Randomised controlled trial; SAFTI: Safety assessment and follow-up telephone intervention; TAU: Treatment as usual; TOC: Teen options for change

\section{Acknowledgements}

We thank the PenCLAHRC Evidence Synthesis Team and Mr Chris Cooper for their support.

\section{Funding}

National Institute for Health Research (NIHR) Collaboration for Leadership in Applied Health Research and Care South West Peninsula (McCabe).

\section{Availability of data and materials}

Not applicable.

\section{Authors' contributions}

$\mathrm{RM}, \mathrm{PX}$ and $\mathrm{RG}$ designed the systematic review. RM, PX, AB screened all records. All authors contributed to the design of the methods and extraction form. RM and PX wrote the initial draft of the paper, which was then revised by $A B$ and $R G$. The final manuscript was read and approved by all authors.

\section{Ethics approval and consent to participate}

This is a review of previously reported studies and required no ethical approval or consent from participants.

\section{Consent for publication}

Not applicable.

\section{Competing interests}

The funder had no role in the design or conduct of the study, collection, analysis, interpretation and management of data, preparation and review of the manuscript, or decision to submit for publication.

\section{Publisher's Note}

Springer Nature remains neutral with regard to jurisdictional claims in published maps and institutional affiliations.

\section{Author details}

'University of Exeter Medical School, Heavitree Road, Exeter EX1 2LU, UK. ${ }^{2}$ European Centre for Environment and Human Health, Knowledge Spa, Royal Cornwall Hospital, Truro TR1 3HD, UK.
Received: 8 February 2018 Accepted: 13 March 2018

Published online: 03 May 2018

\section{References}

1. World health organisation. Suicide: fact sheet. Updated 2017. http://www. who.int/mediacentre/factsheets/fs398/en/. Accessed 24 Apr 2017.

2. Office for National Statistics: Suicides in the UK : 2015 registrations. https:// www.ons.gov.uk/peoplepopulationandcommunity/ birthsdeathsandmarriages/deaths/bulletins/suicidesintheunitedkingdom/ 2015registrations. Accessed 30 May 2017.

3. Gairin I, House A, Owens D. Attendance at the accident and emergency department in the year before suicide: retrospective study. Br J Psychiatry. 2003:183(1):28-33

4. Appleby L, Kapur N, Shaw J, Windfuhr K, Hunt IM, Flynn D, While D, Roscoe A, Rodway C, Ibrahim S et al. The National Confidential Inquiry into Suicide and homicide by people with mental illness annual report 2015: England, Northern Ireland, Scotland and Wales. University of Manchester.

5. Monti K, Cedereke M, Öjehagen A. Treatment attendance and suicidal behavior 1 month and 3 months after a suicide attempt: a comparison between two samples. Archives of Suicide Research. 2003;7(2):167-74.

6. Inagaki M, Kawashima Y, Kawanishi C, Yonemoto N, Sugimoto T, Furuno T, Ikeshita K, Eto N, Tachikawa H, Shiraishi Y, Yamada M. Interventions to prevent repeat suicidal behavior in patients admitted to an emergency department for a suicide attempt: a meta-analysis. J Affect Disord. 2015 Apr 1;175:66-78.

7. Mann JJ, Apter A, Bertolote J, Beautrais A, Currier D, Haas A, Hegerl U, Lonnqvist J, Malone K, Marusic A, Mehlum L. Suicide prevention strategies: a systematic review. JAMA. 2005;294(16):2064-74.

8. Hawton K, Witt KG, Salisbury TL, Arensman E, Gunnell D, Hazell P, Townsend E, van Heeringen K. Psychosocial interventions following self-harm in adults: a systematic review and meta-analysis. Lancet Psychiatry. 2016;3(8):740-50.

9. Da Cruz D, Pearson A, Saini P, Miles C, While D, Swinson N, Williams A, Shaw J, Appleby L, Kapur N. Emergency department contact prior to suicide in mental health patients. Emerg Med J. 2011;28(6):467-71.

10. O'Connor RC, Ferguson E, Scott F, Smyth R, MCDaid D, Park AL, Beautrais A, Armitage CJ. A brief psychological intervention to reduce repetition of self-harm in patients admitted to hospital following a suicide attempt: a randomised controlled trial. The Lancet Psychiatry. 2017;4(6):451-60.

11. Milner AJ, Carter G, Pirkis J, Robinson J, Letters SMJ. Green cards, telephone calls and postcards: systematic and meta-analytic review of brief contact interventions for reducing self-harm, suicide attempts and suicide. $\mathrm{Br}$ J Psychiatry. 2015;206(3):184-90.

12. Hsiao RC, Walker LR. Substance use disorders: part I, an issue of child and adole scent psychiatric clinics of North America, E-book. Elsevier Health Sci. 2016;25(3).

13. Miller WR, Rollnick S. Motivational interviewing: preparing people to change addictive behavior. New York. London: Guilford Press; 1991.

14. Kaner EF, Beyer F, Dickinson HO, Pienaar E, Campbell F, Schlesinger C, et al. Effectiveness of brief alcohol interventions in primary care populations. Cochrane Database Syst Rev. 2007;2:CD004148.

15. McCabe R, Garside R, Backhouse A, Xanthopoulou P. Effective communication in eliciting and responding to suicidal thoughts: a systematic review protocol. Systematic reviews. 2016;5(1):31.

16. Moher D, Shamseer L, Clarke M, Ghersi D, Liberati A, Petticrew M, Shekelle P, Stewart LA. Preferred reporting items for systematic review and metaanalysis protocols (PRISMA-P) 2015 statement. Systematic reviews. 2015;4(1)

17. Beck AT, Kovacs M, Weissman A. Assessment of suicidal intention: the scale for suicide ideation. J Consult Clin Psychol. 1979;47(2):343-52.

18. Butler AM, Malone K. Attempted suicide v. Non-suicidal self-injury: behaviour, syndrome or diagnosis? Br J Psychiatry. 2013;202(5):324-5.

19. Boudreaux ED, Camargo CA, Arias SA, Sullivan AF, Allen MH, Goldstein AB, Manton AP, Espinola JA, Miller IW. Improving suicide risk screening and detection in the emergency department. Am J Prev Med. 2016;50(4):445-53.

20. Critical Appraisal Skills Programme. CASP (insert name of checklist i.e. Systematic Review) Checklist [online]. Available at: http://www.casp-uk.net/ casp-tools-checklists. Accessed 20 Apr 2015.

21. Popay J, Roberts H, Sowden A, Petticrew M, Arai L, Rodgers M, Britten N, Roen $K$, Duffy S. Guidance on the conduct of narrative synthesis in systematic reviews. A product from the ESRC methods programme Version. 2006;1:b92.

22. Miller IW, Camargo CA, Jr., Arias SA, Sullivan AF, Allen MH, Goldstein AB, Manton AP, Espinola JA, Jones R, Hasegawa K et al: Suicide prevention in an 
emergency department population: the ED-SAFE study. JAMA Psychiatry 2017; 74(6):563-570.

23. Fleischmann A, Bertolote JM, Wasserman D, De Leo D, Bolhari J, Botega NJ, De Silva D, Phillips M, Vijayakumar L, Värnik A, et al. Effectiveness of brief intervention and contact for suicide attempters: a randomized controlled trial in five countries. Bull World Health Organ. 2008;86:703-9.

24. Gysin-Maillart A, Schwab S, Soravia L, Megert M, Michel KA. Novel brief therapy for patients who attempt suicide: a 24-months follow-up randomized controlled study of the attempted suicide short intervention program (ASSIP). PLoS medicine. Public Libr Sci. 2016;13(3):e1001968.

25. King CA, Gipson PY, Horwitz AG, Opperman KJ. Teen options for change: an intervention for young emergency patients who screen positive for suicide risk. Psychiatr Serv. 2015;66(1):97-100.

26. Bridge JA, Horowitz LM, Campo JV. Ed-safe_can suicide risk screening and brief intervention initiated in the emergency department save lives? JAMA Psychiatry. 2017;74(6):555-6.

27. Hassanzadeh M, Khajeddin N, Nojomi M, Fleischmann A, Eshrati T. Brief intervention and contact after deliberate self-harm: an Iranian randomized controlled trial. Iranian Journal of Psychiatry and Behavioral Sciences. 2010; 4(2):5-12.

28. Gysin-Maillart AC, Soravia LM, Gemperli A, Michel K. Suicide ideation is related to therapeutic alliance in a brief therapy for attempted suicide. Arch Suicide Res. 2017;21(1):113-26.

29. Pawson R, Tilley N. Realistic evaluation. London: Sage; 1997.

30. Mohr DC, Hart SL, Julian L, Catledge C, Honos-Webb L, Vella L, Tasch ET. Telephone-administered psychotherapy for depression. Arch Gen Psychiatry. 2005;62(9):1007-14.

31. Cooper J, Steeg S, Gunnell D, Webb R, Hawton K, Bennewith O, House A, Kapur N. Variations in the hospital management of self-harm and patient outcome: a multi-site observational study in England. J Affect Disord. 2015; 174:101-5.

32. Stanley B, Brown GK. Safety planning intervention: a brief intervention to mitigate suicide risk. Cogn Behav Pract. 2012;19(2):256-64.

33. Vaiva G, Ducrocq F, Meyer P, Mathieu D, Philippe A, Libersa C, Goudemand $\mathrm{M}$. Effect of telephone contact on further suicide attempts in patients discharged from an emergency department: randomised controlled study. BMJ. 2006;332(7552):1241-5.

\section{Submit your next manuscript to BioMed Central and we will help you at every step:}

- We accept pre-submission inquiries

- Our selector tool helps you to find the most relevant journal

- We provide round the clock customer support

- Convenient online submission

- Thorough peer review

- Inclusion in PubMed and all major indexing services

- Maximum visibility for your research

Submit your manuscript at www.biomedcentral.com/submit

) Biomed Central 